Check for updates

Cite this: Phys. Chem. Chem. Phys., 2020, 22, 20253

Received 16th July 2020,

Accepted 26th August 2020

DOI: $10.1039 / \mathrm{d} 0 \mathrm{cp} 03787 \mathrm{~g}$

rsc.li/pccp

\section{Nanolubrication in deep eutectic solvents}

\author{
James E. Hallett, (D) Hannah J. Hayler (D) and Susan Perkin (D) *
}

We report surface force balance measurements of the normal surface force and friction between two mica surfaces separated by a nanofilm of the deep eutectic solvent ethaline. Ethaline, a 1:2 mixture of choline chloride and ethylene glycol, was studied under dry conditions, under ambient conditions and with added water, revealing surface structural layers and quantised frictional response highly sensitive to water content, including regions of super-lubric behaviour under dry conditions and with added water. We also report exceptionally long-ranged electrostatic repulsion far in excess of that predicted by Debye-Hückel theory for a system with such high electrolyte content, consistent with previously reported observations of "underscreening" in ionic liquid and concentrated aqueous electrolyte systems [Smith et al., J. Phys. Chem. Lett., 2016, 7(12), 2157].

\section{Introduction}

Deep eutectic solvents (DESs) are a class of liquid of growing importance, and are often described as a sub-category or new class of ionic liquids (ILs). ${ }^{1}$ Like ILs, they are a liquid mixture of binary ion pairs with no neutral solvent. Unlike ILs, which are primarily composed of discrete anions and cations of one particular species, DESs contain a complex ionic component and can contain a variety of ionic species. ${ }^{2}$ The term deep eutectic solvent originates from the low melting temperature $T_{\mathrm{M}}$ obtained at the eutectic composition. ${ }^{3}$ DESs are obtained by mixing a hydrogen bond-capable salt (typically a quaternary ammonium salt) with a metal salt or hydrogen bond donor species (HBD). In many cases these two components are crystalline solids at room temperature, whilst the mixture formed is liquid under ambient conditions and with relatively low $T_{\mathrm{M}}$. Furthermore, a wide range of biodegradable, inexpensive components such as amino acids and sugars can be used to form DESs, ${ }^{4,5}$ and it is this flexibility that makes them such an exciting prospect as a green solvent. ${ }^{2}$ Indeed, deep eutectic solvents have been proposed as environmentally friendly alternatives for processes including electrodeposition, ${ }^{6}$ metal oxide processing, $^{7}$ organic synthesis, ${ }^{8}$ and the purification and manufacture of biodiesel. ${ }^{9,10}$

Perhaps the most commonly studied DESs are derived from mixing choline chloride with urea, ethylene glycol or glycerol (so-called reline, ethaline and glyceline) and it is these systems that have been used to make general observations of the unique supramolecular structure of DES systems. It is generally accepted that DESs form due to charge delocalization between

Physical and Theoretical Chemistry Laboratory, University of Oxford,

South Parks Road, Oxford, OX1 3QZ, UK. E-mail: susan.perkin@chem.ox.ac.uk the salt anion and hydrogen bond donor, ${ }^{4}$ but the structural consequences of this unique formation mechanism are still an area of active investigation. Recent work used a combination of neutron diffraction and atomistic modelling to reveal the liquid structure of reline. Hammond and co-workers ${ }^{11}$ measured neutron diffraction data from isotopically substituted reline samples, combined with empirical potential structure refinement (EPSR) to reveal the 3D structure of the solvent, which, in addition to the predicted hydrogen bond network within the network, also showed significant spatial ordering between all the DES components. Subsequent work unveiled the role of water in the 3D structure of the solvent. ${ }^{12}$ Remarkably, even at $42 \mathrm{wt} \%$ water, the 3D spatial order of the DES was retained, as the water was sequestered into nanostructured domains around the cholinium cations. At higher concentrations the structure was disrupted, and was best described as an aqueous solution of the DES components. Recently the structure of DESs has been directly probed via atomic force microscopy (AFM). The nanostructure of reline, ethaline and glyceline at both graphite ${ }^{13}$ and platinum ${ }^{14}$ substrates has been investigated, and was found to be strongly influenced by surface potential, substrate material and water content. For platinum, at positive potentials, the innermost displaceable layer was of comparable thickness to choline $(\sim 0.45 \mathrm{~nm})$ and was thought to be in contact with an impenetrable chloride Stern layer. At negative potentials, the innermost displaceable layer for reline, ethaline and glyceline was too thin to correspond to any DES component other than the HBD, and was thought to be in contact with an impenetrable choline Stern layer. For graphite, the final displaceable layer corresponded to the HBD at all potentials, except for $-1 \mathrm{~V}$ at which point it displayed the same behaviour as the platinum electrode. The difference was attributed to the atomically smooth graphite 
surface more strongly aligning counterions than the platinum surface. They also noted that the magnitude of the final layer push-through force increased with increasing positive potential. However it should be noted that, as AFM does not provide an absolute measure of separation, the hypothesised near-surface structure was based on geometric arguments of molecular size rather than a definitive measure of the liquid layer thickness. In addition to dry DESs, the influence of added water was also studied for the platinum electrode. ${ }^{14}$ It was shown that added water also increased the push-through force and number of layers at open circuit potential, up to a maximum degree of structuring at $40 \mathrm{wt} \%$ water for reline, $30 \mathrm{wt} \%$ water for glyceline and $50 \mathrm{wt} \%$ water for ethaline. However, application of a surface potential disrupted this structure, which was attributed to a rearrangement of the surface-ion composition, resulting in a surface structure closer to that of a salt solution at a charged interface. AFM measurements have also been performed using alkyl ammonium bromide - glycerol based ionic liquids on mica substrates. ${ }^{15}$ Mica substrates are well known to have a negative surface charge through dissolution of potassium cations, ${ }^{16}$ and the DES structure showed layering with a HBD-enriched near-surface layer consistent with the layering observed for an applied negative potential.

A promising application of deep eutectic solvents is that of a lubricant. In many industrial applications spent oil-based lubricants are environmentally damaging and rarely recycled, ${ }^{17}$ so the prospect of a biodegradeable alternative is an enticing one. ${ }^{18}$ Work to date has prioritised bulk measurements of the tribological properties of choline chloride-based DESs, particularly of sliding steel-steel contacts and comparable friction coefficients to oil-based lubricants has been reported. ${ }^{19}$ More recent work reported better lubrication performance from choline chloride-based DES, and found that adding graphene further reduced the coefficient of friction. ${ }^{20}$ Abbott et $a .^{21}$ reported superior lubrication performance from DESs and from ionic liquids than from base oil for steel-steel contacts. Intriguingly they also reported outstanding corrosion resistance from DESs compared to base oil, when the lubricant had become contaminated with aqueous salt solution, and noted a potential application for DESs as marine lubricants. They propose that the formation of an interfacial barrier by the ionic component is the origin of the enhanced lubrication, and the enhancement is optimal at the eutectic composition, ${ }^{21,22}$ and it is the behaviour of this interfacial layer that we interrogate in this study.

The surface force balance is an ideal instrument to perform measurements of the structure and frictional properties of confined films, including aqueous systems, ${ }^{23}$ alkanes $^{24}$ and ionic liquids. ${ }^{25}$ Of particular interest are ionic liquids, which can form layered structures at charged and uncharged interfaces. ${ }^{26-28}$ The coefficient of friction of the film has been shown to vary with the number of confined molecular layers, leading to so-called "quantised friction". ${ }^{29-31}$ Intriguingly the structure of ionic liquid films can also be manipulated by changing the surface charge, ${ }^{32}$ which as a consequence also acts to change the frictional properties, leading to "switchable superlubricity". ${ }^{33}$ The formation of a robust, low friction surface film has lead to ionic liquids being proposed as a novel lubricant system. ${ }^{34-36}$
In this work, we apply the surface force balance technique to the deep eutectic solvent ethaline, a 1:2 molar mixture of choline chloride and ethylene glycol. We report surface layering consistent with previous AFM measurements, ${ }^{13-15}$ but with greater force resolution enabling the detection of weaker surface layers than previously reported, and anomalous longranged forces consistent with 'underscreening'. We measure the frictional response of the surface layers and report quantised friction in the superlubric regime. We also show that both the surface structure and the frictional response are highly sensitive to water content, either through adsorption of atmospheric humidity or through added water. Finally, we propose molecular ordering and mechanisms to rationalise the reported data.

\section{Experimental setup}

\subsection{Surface force balance}

Experiments were performed using a surface force balance (SFB). White light interferometry is used to determine the film thickness $D$, and normal force, $F_{\mathrm{N}}$, between semi-transparent reflective surfaces separated by a thin liquid film. In this work, the surfaces used were back-silvered mica sheets of equal thickness (typically 2-4 $\mu \mathrm{m}$ ). These mica sheets were glued (silver-side down) on to cylindrical lenses (radius of curvature $\sim 10 \mathrm{~mm}$ ) and mounted in the SFB in a crossed-cylinder geometry (Fig. 1). A droplet of deep eutectic solvent was injected between the two mica surfaces, forming a capillary bridge. White light interferometry was used to determine the thickness of the layer between the two silver mirrors, comprising the two equal-thickness mica sheets and the confined liquid film, with a precision of $0.1 \mathrm{~nm}$. One of the lenses was mounted on a piezoelectric tube, capable of normal and lateral motion. The lenses were mounted across orthogonal leaf springs of known spring constants (shear spring $k_{\mathrm{L}}=388$ or $441 \pm 4 \mathrm{~N} \mathrm{~m}^{-1}$ and normal spring $k_{\mathrm{N}}=141 \pm 3$ or $k_{\mathrm{N}}=2670 \pm 84 \mathrm{~N} \mathrm{~m}^{-1}$ ), such that any induced deflection of the springs by the piezoelectric tube could be used to extract the normal interaction force $F_{\mathrm{N}}$ or the lateral force $F_{\mathrm{L}}$.

For further information, the surface force balance technique is discussed in detail elsewhere. ${ }^{24,38}$

\subsection{Materials}

Mica (ruby muscovite) was of optical grade (S\&J Trading). Mica facets of constant thickness (identified by their single interference colour) were attached to mica substrate and coated with an approximately $40 \mathrm{~nm}$ layer silver by evaporating silver shot (99.9999\%, Alfa Aesar) using an HHV Auto306 (HVV Ltd) thermal evaporator. EPON 1004 (Shell) was used to attach mica facets to fused silica cylindrical lenses.

Anhydrous ethylene glycol (Acros, 99.8\%) and choline chloride (Sigma Aldrich, $\geq 99 \%$ ) were used as received. Ethaline was prepared by mixing choline chloride and ethylene glycol in a 1:2 molar ratio. This mixture was subsequently maintained under a nitrogen atmosphere and stirred overnight at $60{ }^{\circ} \mathrm{C}$ 

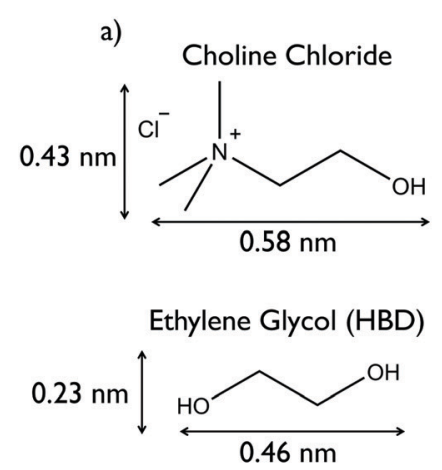
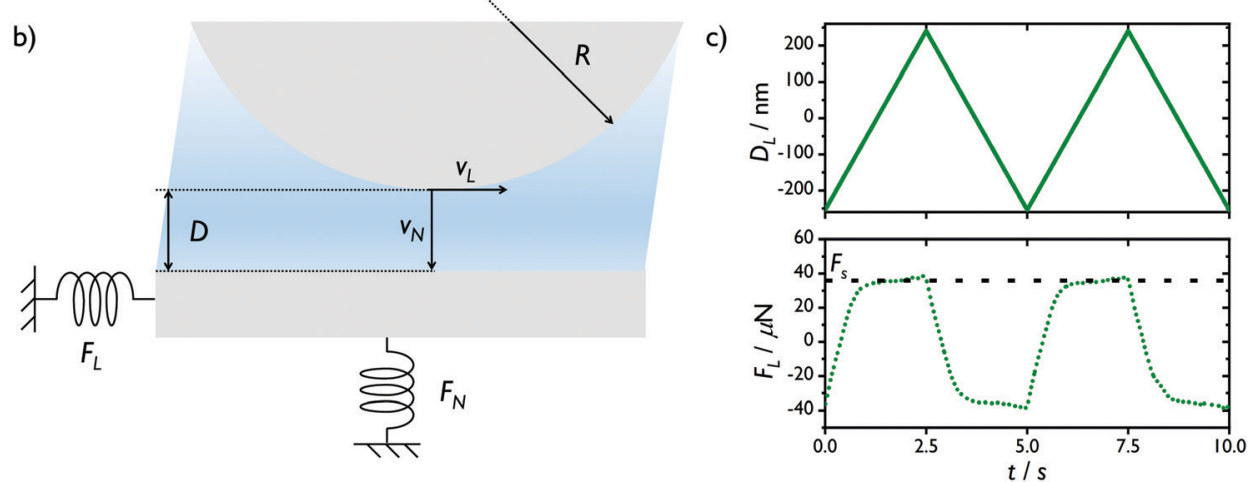

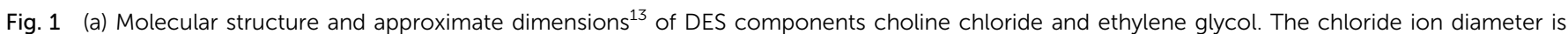

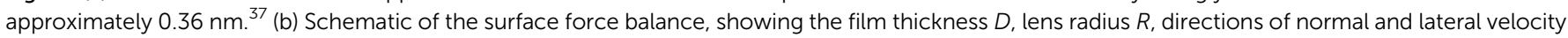

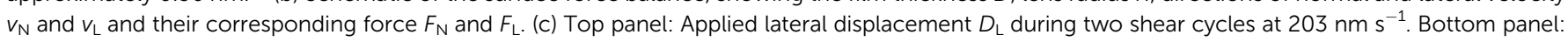
Example of measured lateral friction $F_{\mathrm{L}}$ during shear cycles. The horizontal line indicates the regime of continuous sliding which occurs at $F_{\mathrm{S}}$.

until a homogeneous liquid was obtained. Sample mixtures were subsequently stored under nitrogen until use. Fresh samples were always prepared for use on the following day. Water content of an exemplary ethaline mixture prepared in this way was verified via coulometric Karl Fischer titration (Mettler Toledo) in an argon-filled glovebox, yielding a water content of 19.7 ppm.

All mica cleaving, sample preparation and SFB assembly were carried out in a laminar flow hood (Bassaire P3HF) to maintain dust free conditions, equivalent to ISO 5 class cleanroom environment.

\subsection{Experimental procedure}

Mica-coated lenses were prepared and mounted in the SFB chamber. Prior to sample injection, the thickness of the mica substrates was verified via a contact calibration in air. After retracting the lenses the sample liquid was then injected between the SFB lenses, and the chamber was sealed and purged with nitrogen. An open dish of fresh phosphorous pentoxide powder was also placed in the chamber as a desiccant to maintain dry conditions throughout the measurement. In order to study the role of water in the surface structure and lubrication properties of the confined deep eutectic solvent, water content was controlled by adding water to samples prepared as outlined above, to obtain samples of known water content before injecting the liquid between the lenses.

In a typical measurement mica surfaces are brought together by moving one lens towards the other at fixed rate. At long distance the measured separation decreases linearly. At shorter distances surface forces become measureable and the separation as a function of time deviates from the linear baseline established at long distances. This deviation is then used to extract the normal force. We note that recent work ${ }^{38}$ has shown that viscous drainage of a confined fluid can result in a velocity-dependent hydrodynamic force that can be confused with any long-ranged static surface forces. We confirm that hydrodynamics are not modifying the measured surface force by performing measurements in the regime where the long-range forces have no dependence on approach velocity (typically $\sim 0.5 \mathrm{~nm} \mathrm{~s}^{-1}$ ).

We also perform measurements where the mica surfaces are sheared at fixed rate with a triangle wave function, while simultaneously decreasing their separation (Fig. 1c, top panel). The friction measurements reported here were performed at shearing velocity $v_{\mathrm{s}}=305 \mathrm{~nm} \mathrm{~s}^{-1}$, but were reproducible in the range 101-404 $\mathrm{nm} \mathrm{s}^{-1}$. During these shearing experiments deflection of the shear springs are measured via an air-gap capacitance probe, allowing the lateral force $F_{\mathrm{L}}$ to be determined as a function of time. As the mica layers confine the liquid to a few molecular layers, a measureable stick-slip friction force is measured and we define the kinetic friction force $F_{\mathrm{S}}$ as the lateral force in the regime of continuous sliding (indicated by the dotted line in Fig. 1c, bottom panel). Shear velocities are deliberately much faster than the normal approach velocity, such that a complete shear cycle can be completed at a near constant normal force. We also note that the normal force profiles obtained with and without shear are essentially identical, confirming that shear is not inducing any change in the structure of the surface layers.

\section{Results and discussion}

\subsection{Normal force measurements of ethaline}

In this section we proceed to describe the interfacial structure of ethaline under dry, ambient and hydrated conditions. We present measurements of the normal force, and use molecular size arguments to suggest the arrangement of the confined layers in each system.

3.1.1 Dry ethaline. The normal force profiles obtained for ethaline are shown in Fig. 2. For pure, dry ethaline (2a) the normal force displays two key features. At long range and persisting for tens of nanometres, there is an exponentially decaying long range force (2a inset) of the form $F / R \propto$ $\exp \left(D / \lambda_{\mathrm{s}}\right)$, where $\lambda_{\mathrm{s}}$ is the decay length. By fitting the long range force we obtain a decay length $\lambda_{\mathrm{s}}$ of $12 \pm 1 \mathrm{~nm}$. Pure ethaline is a concentrated electrolyte with a Debye screening length $\lambda_{\mathrm{D}}$ 

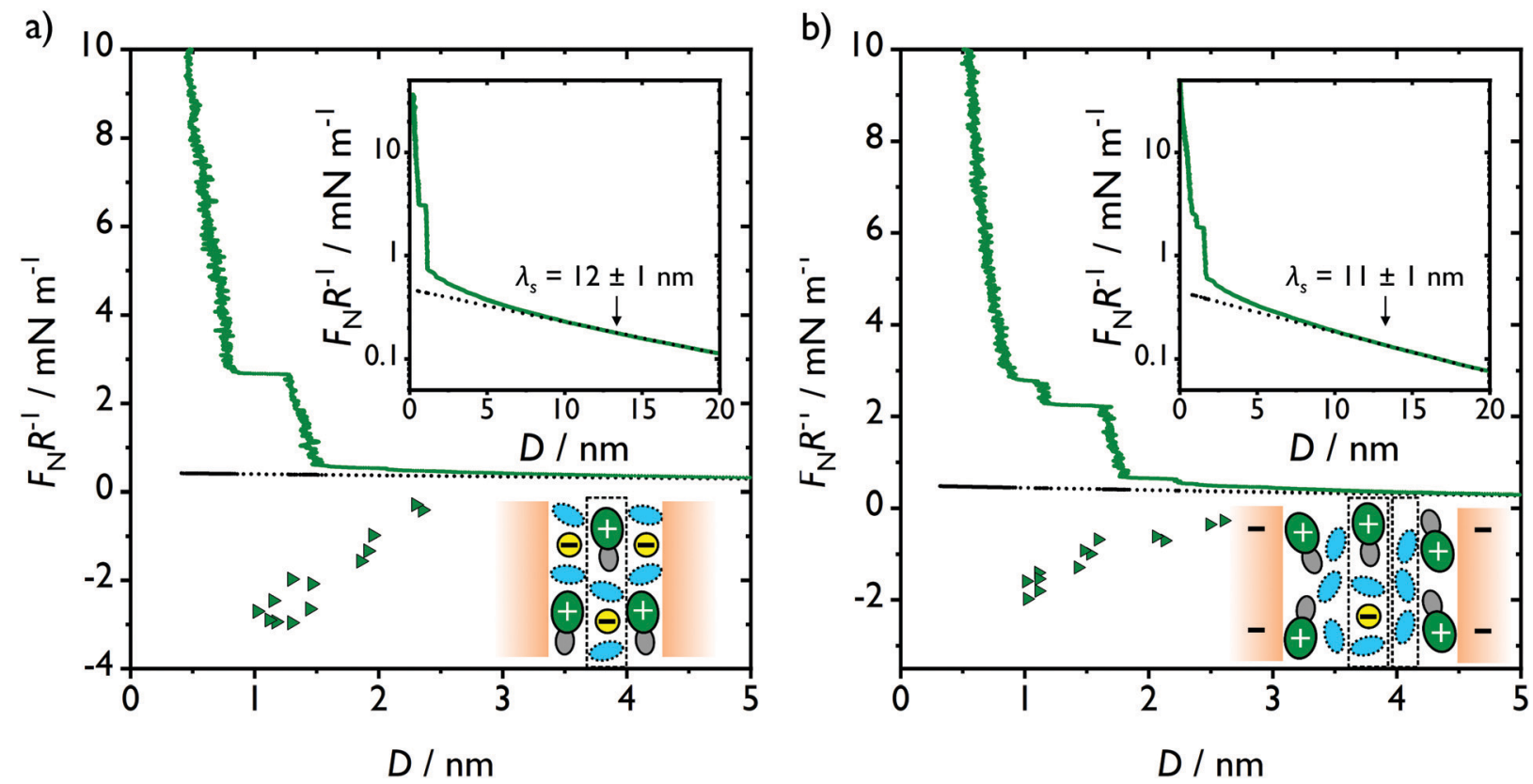

Fig. 2 Force profiles for (a) fresh dry and (b) 24 hour equilibrated ethaline. Triangles indicated retractions from adhesive minima. In both instances the inset shows a log-linear representation of the same data, and the dotted line gives an exponential fit to the long-range force. Radius of curvature $12 \mathrm{~mm}$, mica thickness $3280 \mathrm{~nm}$. Inset cartoons show possible structure, where green cations are choline (with grey hydroxyl groups), yellow anions are chloride, blue ovals are ethylene glycol and the dashed box indicates one detected layer. In (a) the mica is only weakly charged, yielding the so-called "checkerboard" layering. In (b) the magnitude of the charge is greater, resulting in the formation of a choline Stern layer and repelling chloride from the surface layers, resulting in a local excess of ethylene glycol in the form of a HBD layer (smaller dashed box).

of $\sim 0.1 \mathrm{~nm}$ (dielectric constant ${ }^{39}$ and density ${ }^{40}$ taken from literature values), so clearly the measured long-ranged repulsion is not due to conventional Debye-Hückel screening. Concentrated electrolytes have previously been shown to display long ranged repulsions through "underscreening.". ${ }^{41}$ Lee $e t ~ a l .{ }^{42,43}$ proposed a relationship between the measured screening length $\lambda_{\mathrm{s}}$ and the ion diameter $a$ such that $\lambda_{\mathrm{S}} / \lambda_{\mathrm{D}} \sim\left(a / \lambda_{\mathrm{D}}\right)^{3}$. Taking an average ion diameter of $\sim 0.5 \mathrm{~nm}$ as a first approximation, comparable to that measured for reline ${ }^{11}$ and to the size of choline, we would thus expect $\lambda_{\mathrm{S}}=12.5 \mathrm{~nm}$, in very good agreement with the measured value.

At separations smaller than $4 \mathrm{~nm}$, a series of repulsive maxima and attractive minima emerge, which increase in magnitude as the surfaces approach contact. These maxima and minima are measured separately due to the spring-deflection method for detecting force. When $\mathrm{d} F_{\mathrm{N}} / \mathrm{d} D \geq k_{\mathrm{N}}$, there is a spring instability and the surfaces "jump in" to a smaller separation. Similarly individual attractive minima are measured on retraction of the surfaces, which then "jump out" to long distances, the range of which can be used to determine the attractive minima strength. ${ }^{44}$ The oscillatory near-surface equilibrium interaction profile is commonly termed a "structural force", and is the result of sequential layers of molecules being "squeezed out" from between the mica surfaces. The periodicity and decaying amplitude of the structural force can be interpreted to yield information on the molecular arrangement in the liquid film and has been widely studied in a range of solvents, including alkanes ${ }^{45}$ and ionic liquids. $^{34}$

Different approaches have been used to define the layer thickness in confined liquid systems. One approach is to measure the jump in positions from consecutive layers and fit a trendline to define the layer thickness. Recently it was shown $^{38,46}$ that this approach fails to take into account compression of the mica substrates, which is convolved with the layer compression and becomes increasingly pronounced at large normal force. It was also shown that fitting a layer thickness from minima rather than the maxima does not have this issue, as there is no mica compression for negative normal force. An alternative approach is to define the layer thickness as the jump-in distance (supposing an unchanged mica compression during the jump), which yields the same layer thickness as the minima fit. Gooneie et $a l^{47}$ extended this approach by estimating the mica contribution to the layer compression to determine the degree of molecular compression within the film between jumps in. For a DES this compression could be due to a change in molecular order, as for a normal ionic liquid, or through a change in composition in the gap e.g. exclusion or concentration of the HBD. Therefore, we choose to define the step thickness as the jump-in distance, because the assumption that every layer is the same thickness or compresses by the same amount (required to fit a layer thickness as outlined above) might not be true for the multi-component system of a deep eutectic solvent. For instances where the layer thickness appears to be constant, we obtain good agreement between step sizes obtained from the jump-in distance and a fit of the minima.

From the structural force we are able to discern steps of thickness $0.45 \pm 0.03 \mathrm{~nm}$. At least three steps are present and are evidenced in the approach curve and in points which 
indicate the jump out force and distance on retraction. We note that the absolute magnitude of the squeeze-out force showed some variation between experiments of order $50 \%$, which we attribute to small differences in DES composition or degree of mica ionisation, however the layer separations are highly reproducible. Based on dimensional arguments, we expect these steps to correspond to choline ions oriented approximately parallel to the mica surfaces, or ethylene glycol-chloride ion complexes. The orientation of the complexes is less clear due to possible variations in conformation of the ethylene glycol around the chloride. Chloride ions are too small to individually account for the measured step thickness, while surface force measurements of pure ethylene glycol yield steps of thicknesses $\leq 0.4 \mathrm{~nm} .{ }^{13,48}$ For ionic liquids the measured interfacial structure at charged interfaces has been attributed to alternating layers of anions and cations. ${ }^{49,50}$ A measured step thus corresponds to the simultaneous squeeze-out of an anionic layer and a cationic layer, to maintain electroneutrality in the gap and to prevent adjacent layers being of like-charge. This argument rests on step thicknesses measured with SFB and AFM that are too large to solely correspond to one charged species, but can accommodate one layer of each species. ${ }^{34}$ For the measurements reported here the steps are too thin to be due to simultaneous squeeze-out of two oppositely charged layers. Instead, it appears that each layer is of mixed charge, such that only a single molecular layer needs to be squeezed out to maintain electroneutrality in the SFB gap (as opposed to the simultaneous squeeze-out of a cationic layer and an anionic layer). This hypothesised structure is illustrated in Fig. 2a, bottom right. This behaviour has been reported before in so-called mixedlayer "checkerboard" arrangements of ionic liquid cations and anions at interfaces. ${ }^{51-53}$ This hypothesis is supported by recent simulations of the DES reline. Mamme et al. ${ }^{54}$ used molecular dynamics to simulate the structure of reline at neutral and charged interfaces. They found that at negative and positive potentials a Stern layer was present at the interface of choline cations or chloride anions respectively, but beyond this innermost layer all of the other layers were of mixed composition, albeit with some variation in charge density. Intriguingly they also noted that the hydrogen bond donor urea was present in all layers and also had a surface excess for both negative and positive surface potentials. Mamme et al. ${ }^{55}$ also investigated water distribution in reline, which was shown to have a surface excess with applied potential. This supports the mixed-layer hypothesis reported here, although some charge oscillation was reported. From our measurements it is not possible to determine the ion ratios of the squeezed out layers, but we infer that they are electroneutral based on geometrical arguments. However, some charge oscillation in the equilibrium structure could be accommodated during an SFB measurement, whereby electroneutrality in the gap could be conserved by inter-layer exchange of charged species with each other or with smaller, uncharged HBDs (which are present in all layers) during layer squeeze-out. This is supported by recent simulations of shape-asymmetric ionic liquids by Merlet et al. ${ }^{56}$ Mixed composition layers have previously been reported for ionic liquids at uncharged interfaces, ${ }^{57}$ but Merlet et al. showed highly mixed first- and second-layers even with an applied surface potential, and reported "spontaneous exchange of ions between different layers of the electrolyte close to the electrode surface". They attributed this behaviour to voltage-dependent in-plane ordering of the ionic species.

For ethaline that has been allowed to equilibrate with the mica substrates overnight in a dry nitrogen atmosphere (2b) we make two key observations. Firstly, the long range repulsion is still present and of comparable range to the fresh, dry sample. Secondly, we note that the surface layering has changed, and now in addition to the $0.45 \mathrm{~nm}$ steps there is also a $0.23 \pm$ $0.03 \mathrm{~nm}$ step adjacent to the innermost surface layer. The only component in the DES with a spatial dimension small enough to correspond to this step is the HBD ethylene glycol, which is of comparable thickness. We therefore propose that the $0.23 \mathrm{~nm}$ step corresponds to ethylene glycol lying parallel to the mica surface.

We also note that for dry ethaline the final (immovable) layer thickness is $\sim 0.8 \pm 0.05 \mathrm{~nm}$ after the final jump-in, while for equilibrated ethaline (for the same normal force) it is consistently slightly larger $(\sim 0.9 \pm 0.05 \mathrm{~nm})$. Although absolute separation in any surface force balance measurement is susceptible to errors induced by misalignment of the optical path, or from flattening of the mica surfaces with increasing normal force, ${ }^{31,46}$ we note that this supports the idea that the interfacial structure is somehow changing as the system equilibrates.

We now speculate on the origin of the structural transition observed in the equilibrated sample. Mica substrates are well known to develop a negative surface charge through dissolution of potassium cations, ${ }^{16}$ and the DES structure for the equilibrated sample (Fig. 2b) displays layering with a HBD-enriched near-surface layer consistent with the layering observed with $\mathrm{AFM}^{14}$ for an applied negative potential. However, initially this is not the case and no HBD layer is observed. We therefore propose two mechanisms for the formation of the HBD layer in the equilibrated sample. Firstly, the equilibration time for the surface potential of mica is approximately 1 hour in ultrapure water. ${ }^{23}$ It is likely that the equilibration process is slower in the concentrated electrolyte environment of a deep eutectic solvent, so it may take several hours of equilibrating at large separations for the mica to become sufficiently charged for the surfacecholine-HBD structure to form. As the magnitude of the surface charge increases more choline molecules will move to the surface to counter the negative surface charge, and are likely to orient at least somewhat perpendicularly to the negatively charged mica surface, with the choline charged group oriented towards the mica and the hydroxyl group towards the bulk liquid. In doing so they will exclude ethylene glycol which was previously at the surface, forming a HBD-rich layer adjacent to the choline Stern layer. This is illustrated in Fig. 2b, bottom right. One might expect the ethylene glycol layer to lie parallel to the mica surface to maximise hydrogen bonding between their hydroxyl groups and the exposed hydroxyl groups of the interfacial (perpendicularly oriented) choline, which will be of higher density than the parallel-oriented choline of the next 
molecular layer. Secondly, although the SFB chamber is maintained under nitrogen atmosphere with a phosphorous pentoxide dessicant, it is likely that some atmospheric humidity is eventually adsorbed by the hygroscopic DES sample. It is hard to verify if and by how much the water content is changing over the course of a measurement - the volume of liquid held between the mica surfaces (typically $\sim 20 \mu \mathrm{l}$ ) is much too little for Karl Fischer titration. Nevertheless, water is known to change the surface structure of ionic liquids measured with the $\mathrm{SFB}^{29,58-60}$ and so may also play a role in this process as well, either through enhancing the solvation of the surface potassium ions as outlined above, or by changing the inter-molecular interactions such as via enhanced hydrogen bonding. Hammond et $a .^{12}$ note that small amounts of added water enhance the DES structure via strengthening the hydrogen bond network, rather than modifying it to a different structure, and we set about making a comparable measurement here.

In order to test the effect of small quantities of water in the DES, we take a dried sample and allow it to equilibrate with the ambient environment for several hours. Karl Fischer titration was performed on an exemplary sample, yielding a water content of $3800 \mathrm{ppm}$, comparable to values reported for undried DESs. ${ }^{14}$ We then perform measurements in the sealed SFB chamber, recording force profiles at different times after exposing the surfaces to the air-equilibrated liquid.

We immediately note that for this "ambient" sample, the surface layering is notably stronger with identical $0.45 \mathrm{~nm} \pm$ 0.05 step size to the dry case (Fig. 3, 1 hour). This supports the hypothesis that small amounts of water enhance the hydrogen bond network in the liquid, which one would expect to increase the squeeze out force. However, the stronger molecular layering in the humid sample could also be due to enhancement of the mica surface charge via increased dissociation of the potassium ions by absorbed water. A similar dramatic enhancement of the squeeze out force by ambient water has also been reported in ionic liquids. ${ }^{59,60}$ We find that this structure is robust over several hours and many repeat measurements. We also allow the liquid film to equilibrate overnight at large separation and observe an additional structural transition (Fig. 3, 24 hours). It appears that the inner two steps are squeezed out simultaneously at a force between the previous two values, and the thickness of this intermediate step is $0.72 \pm 0.05 \mathrm{~nm}$. We also find more pronounced steps of thickness $\sim 0.7 \mathrm{~nm}$ at longer distances. We note that the longest single molecular component in ethaline is choline, of length $\sim 0.58 \mathrm{~nm}$, therefore the layer thickness must no longer correspond to a single molecular layer. We propose that for sufficiently long equilibration times and sufficient adsorbed water, the mica surfaces are able to become charged enough to separate the counter-ions and co-ions into distinct layers, comparable to the oscillatory charge density structure of a conventional room temperature ionic liquid at a charged mica surface (shown schematically in Fig. 3). For alternating layers of anions and cations, a measured step must be of a cationic layer and an anionic layer to conserve electroneutrality. It is easy to see (Fig. 1a) that a layer of choline molecules oriented parallel to the mica surface, and a mixed

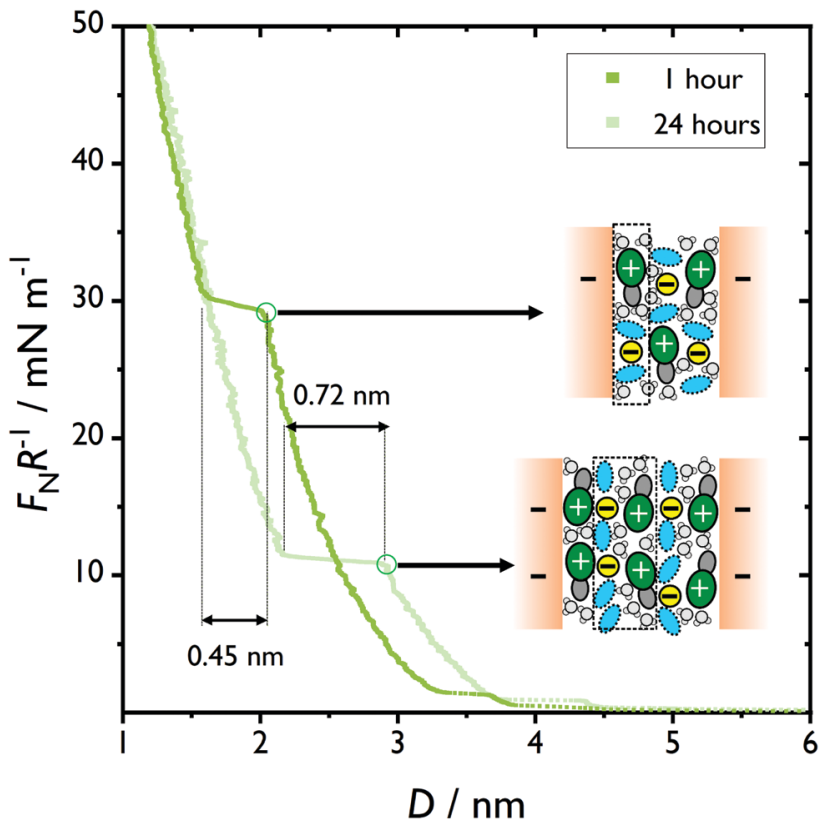

Fig. 3 Force profiles for ambient ethaline at two times: shortly after injecting the liquid ( 1 hour, light green) and after equilibration overnight (24 hours, dark green). The 1 hour profile is representative of runs performed over the first $\sim 10$ hours of measurement. The 24 hours profile was also repeatable over $\sim 10$ hours, which implies some sort of threshold condition was achieved (e.g. sufficient surface charge) between the force profiles rather than a continuous equilibration process. Cartoons are shown to the right illustrating the possible molecular structures in the circled positions in the force profiles. Dotted boxes in the cartoons indicate the squeezed-out layer detected immediately to the left of the circled points. Radius of curvature $8 \mathrm{~mm}$, mica thickness $3153 \mathrm{~nm}$.

layer of ethylene glycol (also oriented parallel to the mica surface) and chloride ions would have a thickness in the range $0.66-0.79 \mathrm{~nm}$, so this is consistent with our hypothesis.

We also note that the layers appear to be highly compressible before finally reaching an instability and squeezing out a layer of molecules. This is consistent with the liquid-phase behaviour of a dilute monolayer, which can undergo compression before squeeze-out, such as through reorganisation of the layer, or by excluding other molecular species - in this instance likely through the exclusion of the uncharged species (ethylene glycol or water).

We therefore make three statements: firstly, that the humid sample is inherently more structured than the dry sample, which at least in part appears to be through internal hydrogen bonding throughout the liquid. Secondly, as for comparable measurements in ionic liquid systems, ${ }^{59}$ trace water enhances the surface charge of the mica by increasing the dissociation of potassium ions from the mica surface. This increase in surface charge is sufficient to rearrange the surface structure of the DESs from a mixed "checkerboard" structure to discrete layers of cations and anions that must be squeezed out simultaneously to maintain electro-neutrality in the gap. Thirdly, uncharged species (water and ethylene glycol) can be squeezed out continuously at the forces applied between steps.

3.1.2 The role of added water in surface structure. In the previous section we explored the normal surface structure of 
dry ethaline, and ethaline with adsorbed ambient water. In this section we explore the structure of ethaline-water mixtures.

The normal force profiles obtained for ethaline with $30 \mathrm{wt} \%$ and $50 \mathrm{wt} \%$ water are shown in Fig. 4 . In both instances we note three significant differences when compared to dry and humid ethaline. At separations greater than $5 \mathrm{~nm}$, a weak, long range repulsive force is measured, the range of which decreases with increasing water content. As in the previous section, we can fit this exponential decay to obtain $\lambda_{\mathrm{s}}$. For $30 \mathrm{wt} \%$ water we obtain a $\lambda_{\mathrm{s}}$ of $6 \pm 1 \mathrm{~nm}$, and for $50 \mathrm{wt} \%$ water we obtain a $\lambda_{\mathrm{s}}$ of $4 \pm 1 \mathrm{~nm}$ (insets, Fig. $4 \mathrm{a}$ and b respectively). For conventional Debye-Hückel screening the opposite trend would be expected, so these measurements also appear to display underscreeninglike behaviour. We expect underscreening to become significant where $\left(a / \lambda_{\mathrm{D}}\right)>1,{ }^{43}$ a condition these state points fulfil so attributing our observation of decreasing screening length with increasing water content to underscreening is appropriate.

At separations smaller than $5 \mathrm{~nm}$ a structural force emerges for both $30 \mathrm{wt} \%$ and $50 \mathrm{wt} \%$ water. We observe a similar structure with at least 3 molecular layers present and of comparable thickness for both concentrations. We measure outer step thicknesses of $0.74 \pm 0.08 \mathrm{~nm}(0.82 \pm 0.03 \mathrm{~nm}$ from jump outs) and $0.80 \pm 0.08 \mathrm{~nm}(0.82 \pm 0.02 \mathrm{~nm}$ from jump outs $)$ for $30 \mathrm{wt} \%$ and $50 \mathrm{wt} \%$ water respectively. As for Fig. 3, the measured step thicknesses for both concentrations are too large to solely be due to a single molecular species, and instead appear to correspond to a pair of oppositely charged molecular layers. This is illustrated in Fig. 4b, bottom right. However, for the innermost measured layer we obtain a smaller step thickness of $0.50 \pm 0.10 \mathrm{~nm}$ and $0.55 \pm 0.10 \mathrm{~nm}$ for $30 \mathrm{wt} \%$ and $50 \mathrm{wt} \%$ water respectively. We also note that the squeeze-out force for the innermost layer is rather larger than for pure ethaline, such that some compression of the mica is necessary (resulting in an underestimation of the film thickness at closest approach, particularly for the thicker mica used for $50 \mathrm{wt} \%$ water) before the surfaces jump together. The adhesive minima are also notably stronger than for dry ethaline, with innermost layer adhesions of $16-20 \mathrm{mN} \mathrm{m}^{-1}$ for both $30 \mathrm{wt} \%$ and $50 \mathrm{wt} \%$ water. Previous reports of the adhesion of mica in pure water give a value of $15 \pm 3 \mathrm{mN} \mathrm{m}^{-1},{ }^{23}$ in good agreement with the values reported here. The observation of mica adhesion comparable to values reported for pure water, combined with the final step thickness of $0.55 \pm 0.10 \mathrm{~nm}$, is consistent with the picture of a Stern layer of perpendicularlyoriented choline ions being excluded from the film as e.g. the surface charge is regulated at such close confinement, leaving only a surface film of water ions in the gap. The enhancement of interfacial structure in ethaline by added water has been reported previously, but we also note some differences between our data and previous AFM measurements. ${ }^{14}$ At OCP two or three pronounced steps of thickness $\sim 1 \mathrm{~nm}$ were measured, but at negative applied potentials they reported that the layers in the interfacial structure measured at OCP "almost disappear when a potential is applied and are replaced with a short-range exponential decay".
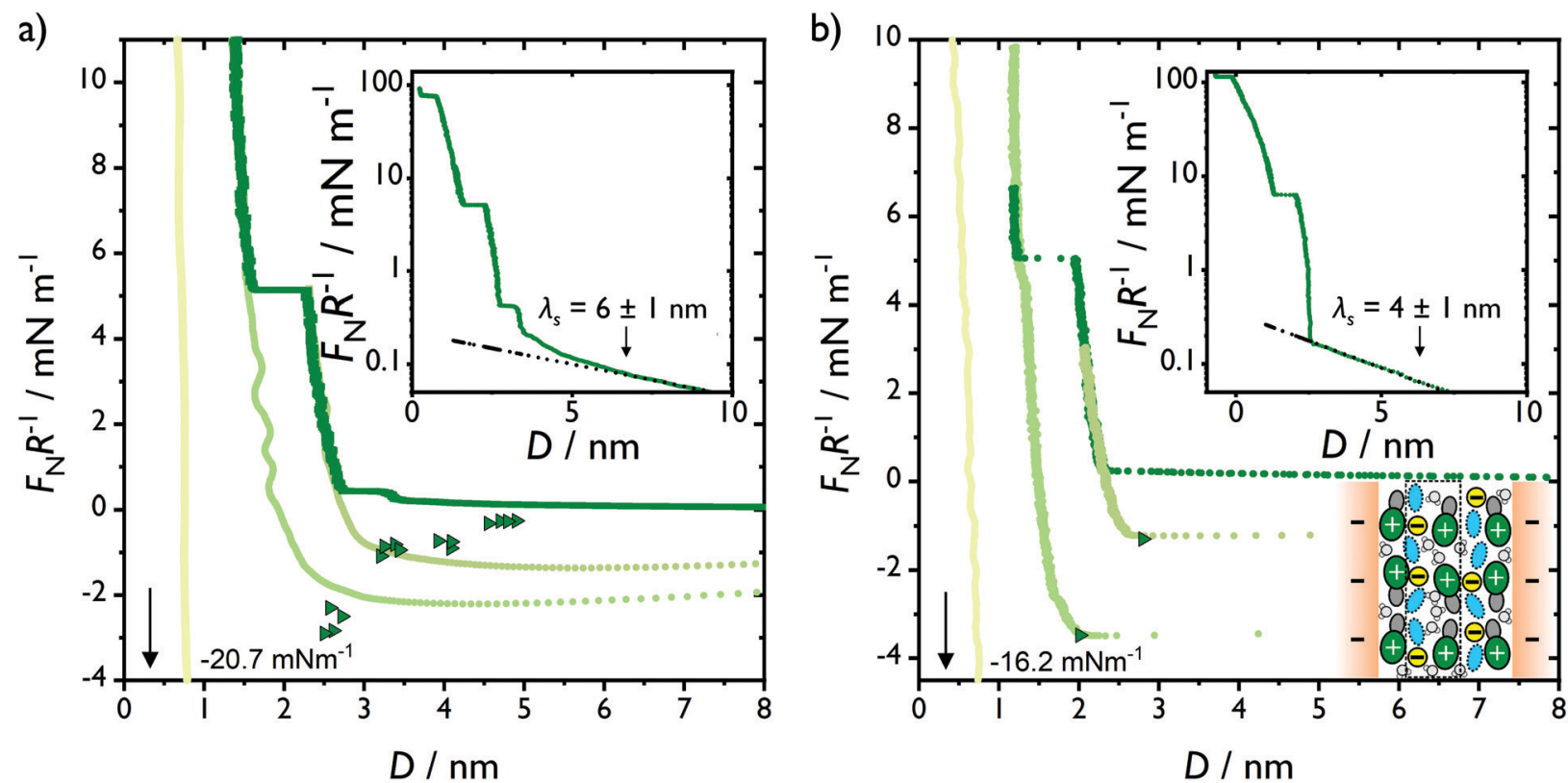

Fig. 4 Force profiles (dark green) for ethaline with (a) 30 wt\% and (b) 50 wt\% water continuous retractions (light greens) from different layers are shown to indicate adhesive minima. In both instances the inset shows a log-linear representation of a comparable measurement at the same concentration, and the dotted line gives an exponential fit to the long-range force. Radius of curvature (a) $11 \mathrm{~mm}$ (inset $12 \mathrm{~mm}$ ) and (b) $8 \mathrm{~mm}$ (inset $10 \mathrm{~mm}$ ). Mica thickness (a) $2388 \mathrm{~nm}$ (inset $5368 \mathrm{~nm}$ ) (b) $2381 \mathrm{~nm}$ (inset $8032 \mathrm{~nm}$ ). Inset cartoon in (b) shows possible structure of both hydrated films, following colouring convention of Fig. 2. Added water increases the magnitude of the mica surface charge and modifies the interactions between the DES components, resulting in alternating charged layers, such that a squeezed-out layer (shown in the dashed box) includes an anionic and a cationic layer to maintain electroneutrality in the film. Step thicknesses imply a similar structure for $30 \mathrm{wt} \%$ and $50 \mathrm{wt} \%$ water, but the greater persistence of layers for $30 \mathrm{wt} \%$ suggests that for $50 \mathrm{wt} \%$ the extra water molecules act to disrupt the DES structure further from the mica surface. 
How can we consolidate these results? We note that the confined layers display a compression of around $0.1-0.3 \mathrm{~nm}$ before they are squeezed out. AFM approach speeds are typically at least an order of magnitude quicker than an equivalent SFB measurement, ${ }^{15}$ so are less sensitive to any slow rearrangements in the confined film and so could also result in a larger layer thickness. The interfacial structure of an electrolyte is intimately linked to the roughness, crystallinity and electrical (i.e., conductor or dielectric) properties of the surface. ${ }^{49,57}$ Furthermore, the local electrolytic environment experienced by an AFM tip can more rapidly change when a potential is applied (through flux of counter- and co-ions to and from the bulk ${ }^{14}$ ), such that an AFM measurement is not necessarily representative of the native interfacial structure. On the other hand a surface force balance measurement has a contact area several orders of magnitude larger, and so is both more representative of the native interfacial structure and less prone to short-time diffusive processes altering the apparent structure. We also note that differences in the interfacial structure of ionic liquids, measured with AFM and SFB, have been reported within the same manuscript. ${ }^{59}$

\subsection{Frictional response}

We now present measurements of the shear response of confined dry and wet ethaline films which we measure as a function of varying load.

3.2.1 Dry ethaline. We first investigate the frictional response of confined dry ethaline films. Fig. 5a shows the film thickness $D$ and the kinetic friction force $F_{\mathrm{S}}$, both as a function of the normal force $F_{\mathrm{N}}$ for dry ethaline. At long distances (or equivalently negligible normal force) the shear force is essentially zero, as there is no transmission of lateral force through the bulk liquid. However, it is clear that as the normal force $F_{\mathrm{N}}$ is increased and molecular layers are squeezed out of the film (evidenced by a step in the thickness $D$ ), there is a simultaneous step in both the magnitude (corresponding to a transition from liquid-like to increasingly solid-like behaviour) and more significantly, the gradient of $F_{\mathrm{S}}$. Within each layer the gradient $F_{\mathrm{S}} / F_{\mathrm{N}}$ is quasi-linear, yielding the coefficient of friction $\mu$. This "quantised friction" has previously been reported for $1: 1$ and $2: 1$ ionic liquids. $^{28,60,61}$ For dry ethaline the coefficient of friction of the confined molecular layers is superlubric $(\mu<0.01)$ at all measured loads. One can also obtain a value for the contact pressure $\sigma_{\mathrm{c}}$ during the friction measurement using the Johnson-Kendall-Roberts (JKR) model for contact mechanics, which provides an estimate of the contact area for modest $\left(<4 \mathrm{mN} \mathrm{m}^{-1}\right)$ normal force. ${ }^{24}$ We note that this force is not uniform over the contact region and becomes less appropriate at larger normal force as the surfaces become more deformed, ${ }^{46}$ therefore we only report the contact pressure for the first (outermost) solid-like layer in the shear force for each sample. For the dry case we find $\sigma_{\mathrm{c}}=23 \pm 5 \mathrm{kPa}$. Although the contact pressure is comparable to the fresh sample $\left(\sigma_{\mathrm{c}}=25 \pm 5 \mathrm{kPa}\right)$, after equilibration overnight the frictional response changes considerably. We noted earlier (Fig. 2b) that equilibration leads to the formation of a HBD-rich layer adjacent to a tightly-bound Stern layer. In the outermost measured molecular layers the coefficient of friction is still superlubric, but is remarkably different for the HBD and innermost layer. For the HBD layer the coefficient of friction increases markedly to $0.03 \pm 0.01$, and for the innermost layer it increases further to $0.12 \pm 0.02$. These values are comparable to those obtained previously for ionic liquids, ${ }^{28,60,61}$ but represents a change of more than a factor of 20 for the innermost layer compared to the dry case. These values compare favourably to bulk measurements of the friction coefficient of ethaline reported previously,
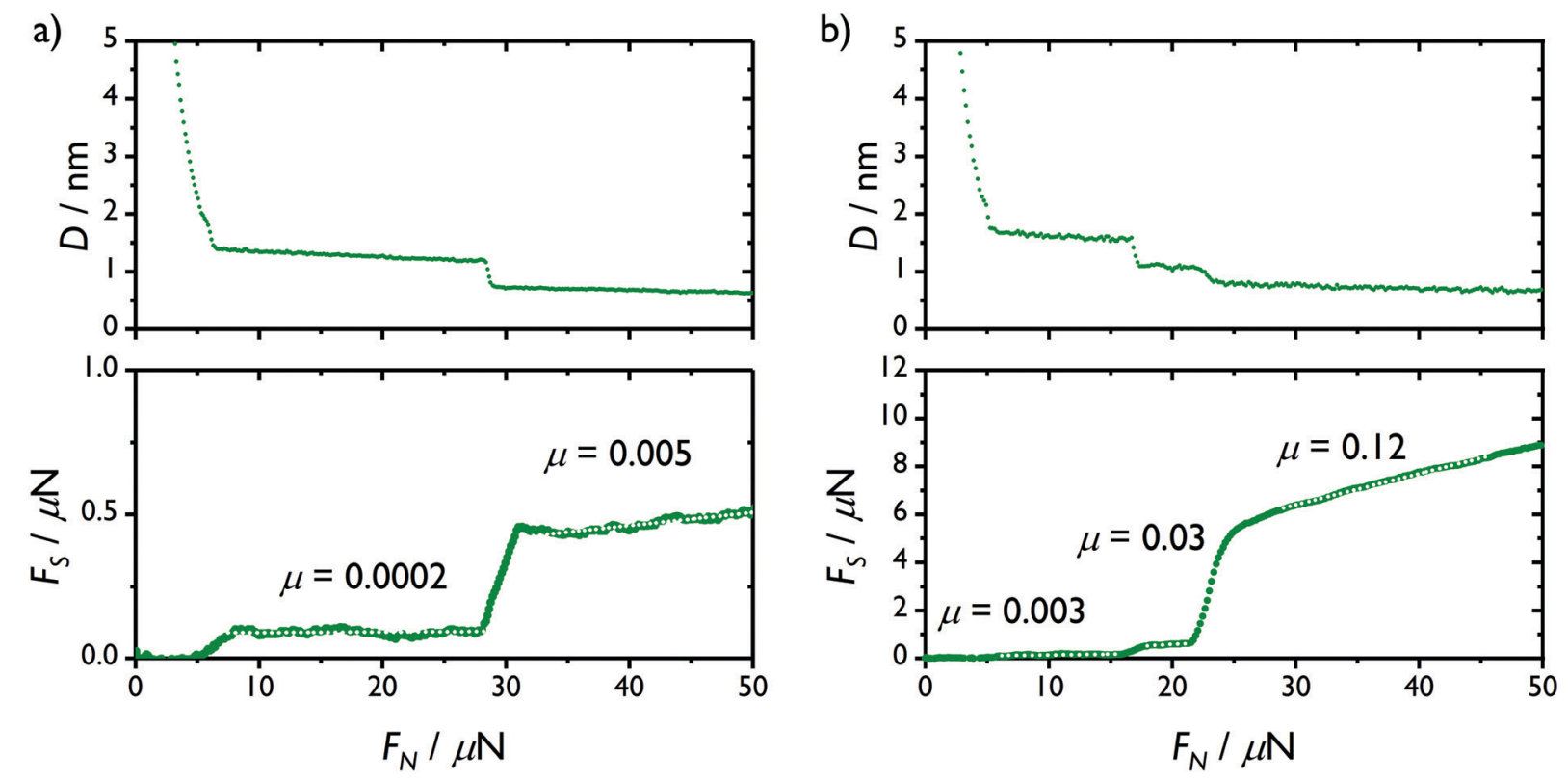

Fig. 5 Film thickness $D$ and shear force $F_{\mathrm{S}}$ as a function of normal force $F_{\mathrm{N}}$ for (a) fresh dry and (b) 24 hour equilibrated ethaline. Coefficients of friction are shown for white-dotted quasi-linear regions. Radius of curvature $8 \mathrm{~mm}$, mica thickness $3280 \mathrm{~nm}$. 
which vary between $0.02^{20}$ and $0.6^{21}$ depending on method, material and surface roughness. We propose that the improved performance reported here is due to the increased flatness of mica when compared to e.g. steel-steel contacts. While a rough surface has a reduced real contact area, the roughness also disrupts any long range ordered surface structure. By using atomically flat mica as a substrate, the surface layering can propagate over larger lengthscales, allowing continuous interlayer sliding over a wide area. The data shown is representative of multiple repeats and we obtain a qualitatively similar quantised friction response during separate experiments, but the calculated friction coefficients can vary by up to factor of 2 , which we attribute to small differences in composition (both ethylene glycol: choline chloride ratio and adsorbed water), crystallograpic misalignment of the mica substrates and variations in the precise contact geometry. A similar increase of friction coefficient with equilibration (but without structural transformation in the normal force) has been reported for dodecane $^{45}$ and ionic liquid ${ }^{29}$ equilibrated against humid air. For dodecane the increase in friction coefficient with water content was attributed to the formation of insoluble crystalline salt bridges, which is unlikely to be the case here. On the other hand, the increase in friction coefficient for the ionic liquid was attributed to localisation of water molecules at the charged IL headgroups, and subsequent solidification of the film, which acts to increase the force required for adjacent layers to slide past each other. This hypothesis is more compatible with the DES system reported here. As water is accommodated in the nanostructure at the interface, it appears that either the active shear plane is changing, or that it is altered in such a way that sliding is resisted. Ashworth et al. ${ }^{62}$ reported many competing hydrogen-bond interactions in DESs - forming a so-called "alphabet soup". Trace amounts of water can further enhance the hydrogen bond network in the DES ${ }^{12}$ and so an increase in friction coefficient could be the result of enhanced inter-layer hydrogen bonding, which acts to resist sliding.

3.2.2 Ethaline-water mixtures. We now proceed to investigate the frictional properties of ethaline-water mixtures. In any real world application of ethaline as a lubricant contamination by water would be unavoidable, and so it is essential to determine to what extent the frictional properties can change. Fig. 6a and $\mathrm{b}$ show the film thickness $D$ and the kinetic friction force $F_{\mathrm{S}}$, both as a function of the normal force $F_{\mathrm{N}}$ for ethaline with $30 \mathrm{wt} \%$ and $50 \mathrm{wt} \%$ water respectively. We report friction coefficients over a comparable range of normal force to the ethaline data (6, lower panels) and also with a substantially greater normal force $(6$, inset, lower panels) in order to access the innermost layer of the force profile. Although their normal force profiles are qualitatively similar (Fig. 4a and b) and their contact pressures are comparable $\left(\sigma_{\mathrm{c}}=20 \pm 3 \mathrm{kPa}\right.$ and $\sigma_{\mathrm{c}}=16 \pm 3 \mathrm{kPa}$ for $30 \mathrm{wt} \%$ and $50 \mathrm{wt} \%$ respectively), we report dramatic differences in frictional response of ethaline with $30 \mathrm{wt} \%$ and $50 \mathrm{wt} \%$ water. For $30 \mathrm{wt} \%$ water we are able to resolve two layers of distinct friction coefficient: an outer layer with $\mu=0.0002 \pm 0.0001$, and an inner layer with $\mu=0.1 \pm 0.02$. During this step to the inner layer there is also a marked increase in $F_{\mathrm{S}}$, indicative of liquid-to-solid transition upon confinement. ${ }^{24}$ This is comparable to the behaviour of the equilibrated ethaline sample with no added water. In the force profile there is a further outer layer but the friction coefficient is below the detection threshold for this measurement. The inner friction layer corresponds to the second layer in the normal force profile. When the innermost layer is reached in the sheared approach, the kinetic friction force $F_{\mathrm{S}}$ exceeds $\sim 110 \mu \mathrm{N}$ (Fig. 6a and b, bottom panel insets), at which point the lateral force varies directly with the applied shearing amplitude and displays a triangle wave shape (Fig. 1c, top panel). The triangular shape is indicative of rigid coupling such that there is no relative lateral motion between the driving and responding substrates. It is likely that $F_{\mathrm{S}}$ will continue to increase in a linear manner within the same layer, but we are unable to access lateral forces in excess of $\sim 110 \mu \mathrm{N}$ to reach continuous sliding, while also approaching with the piezotube in our current setup. The innermost layer displays rigid coupling during applied shear until a significant negative normal force is applied, shortly before the surface jumps out. From this we can estimate the friction coefficient $\mu=2.5 \pm 1.0$. For $50 \mathrm{wt} \%$ water we are also able to resolve two layers of distinct friction coefficient, but here the friction coefficient is significantly lower than for $30 \mathrm{wt} \%$ and maintains superlubricity up to substantial normal force. The outer layer has a friction coefficient $\mu$ in the range 0.0002-0.002, while the inner layer has a friction coefficient of $\mu=0.008 \pm$ 0.002. During this step to the inner layer there is also much weaker increase in $F_{\mathrm{S}}$ compared to $30 \mathrm{wt} \%$. We discuss the physical origin of this difference in the following section but we also note that $F_{\mathrm{S}}$ scales with the surface area of the contact spot, which can vary between measurements due to e.g. variations in curvature at and away from the apex, or deviations from the crossed-cylinder geometry. Previous works on the lubricative properties of alkanes have shown differences in the yield point that vary by as much as a factor of 5 for the same solven $t^{45}$ but crucially the friction coefficient remains the same. A further outer layer was detected in the normal force profile (Fig. $4 \mathrm{~b}$ ), but the friction coefficient was below the detection threshold for this measurement. As for $30 \mathrm{wt} \%$ water, we are also able to access the transition to the innermost layer, but again measure rigid coupling. By applying a negative normal force we can access sliding before the surfaces jump out, yielding a friction coefficient of $\mu=3.0 \pm 1.0$. As with pure ethaline, the coefficient of friction measured for ethaline with added water compares favourably to the values reported previously, ${ }^{19,20}$ supporting the assertion that DESs could have application as marine lubricants. ${ }^{21}$

3.2.3 Structural origin of lubrication. In this section we discuss possible mechanisms behind behaviour observed for differently-prepared ethaline. In the previous sections we showed that a superlubric regime can be accessed even under considerable normal force for ethaline with $50 \mathrm{wt} \%$ water. This is remarkable in that, for humid ethaline and ethaline with $30 \mathrm{wt} \%$ water, the friction coefficient was significantly higher than for superlubric dry ethaline, yet for $50 \mathrm{wt} \%$ water the frictional response is remarkably similar to the dry case. How can we rationalise this unusual re-entrant superlubric 
a)
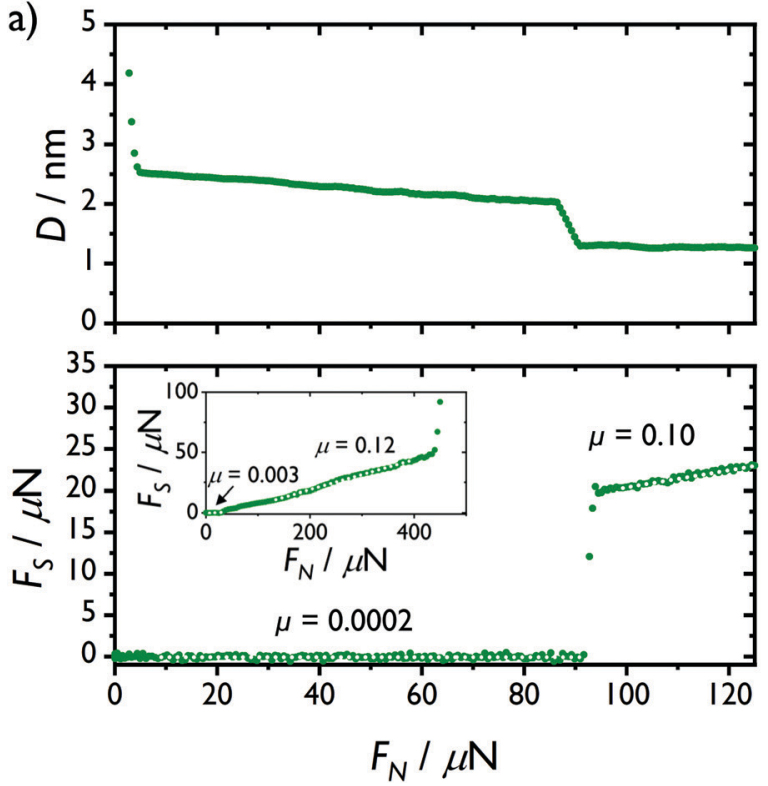

b)
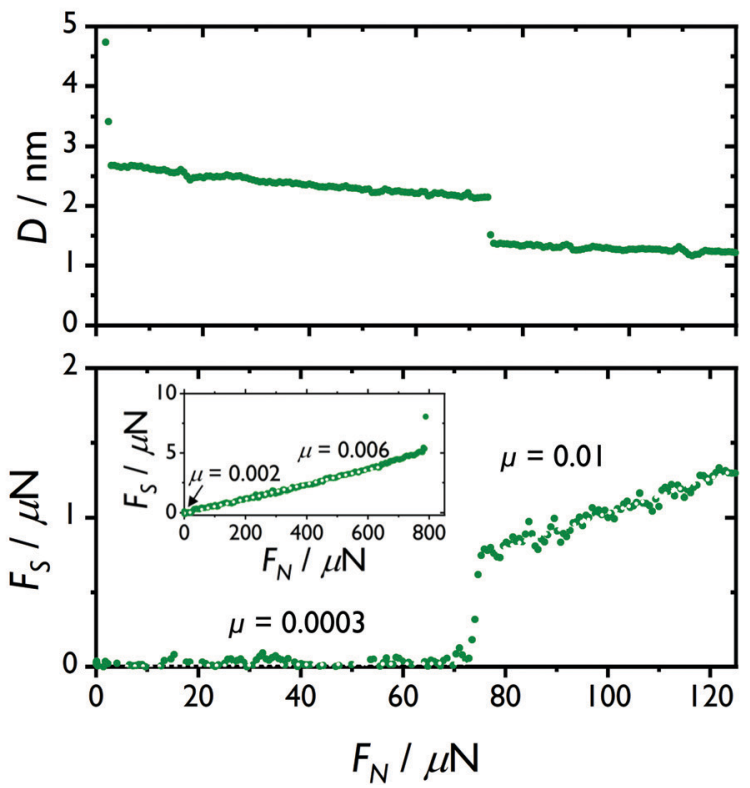

Fig. 6 Film thickness $D$ and shear force $F_{\mathrm{S}}$ as a function of normal force $F_{\mathrm{N}}$ for ethaline solutions with (a) 30 wt\% and (b) 50 wt\% water. Coefficients of friction are shown for white-dotted quasi-linear regions. Insets show measurements with a stiffer normal spring, which show the reproducibility of the frictional response for repeat measurements and at much greater normal force. Radius of curvature (a) $10 \mathrm{~mm}$ and (b) $8 \mathrm{~mm}$. Mica thickness (a) $4261 \mathrm{~nm}$ and (b) $2381 \mathrm{~nm}$. Insets - Radius of curvature (a) $12 \mathrm{~mm}$ and (b) $10 \mathrm{~mm}$. Mica thickness (a) $2388 \mathrm{~nm}$ and (b) $8032 \mathrm{~nm}$.

behaviour with added water? Neutron diffraction measurements from reline ${ }^{12}$ show an enhancement in the hydrogen bond network for small amounts of added water ( $\leq 6 \mathrm{wt} \%)$. This enhanced hydrogen bonding could play a role in the transition from superlubric to IL-like frictional response displayed in equilibrating ethaline, such as by resisting the movement of adjacent molecular layers, or could result in a different sliding plane as the surface structure changes during the formation of the HBD layer. Neutron diffraction measurements from reline also show that the DES structure is fundamentally retained even up to $42 \mathrm{wt} \%$ water, as $\mathrm{H}_{2} \mathrm{O}$ is sequestered into nanostructured domains around the cholinium cations. In such a system the sliding plane could therefore remain unchanged with added water, reflecting the comparable friction coefficients from equilibrated ethaline and $30 \mathrm{wt} \%$ water. The disruption of the DES structure beyond $42 \mathrm{wt} \%$ water could therefore result in the formation of a new sliding interface at comparable pressure with a much lower friction coefficient, as reported here for $50 \mathrm{wt} \%$ water. The observation of a re-entrant superlubric regime provides intriguing supporting evidence for a structural transition with added water, resulting in nanoscale phase seperation, as excess water is no longer fully accommodated within the existing DES framework. Instead, the formation of e.g. segregated water layers or water rich domains, with enhanced in-plane fluidity, results in new sliding interfaces that reflect the increasing dynamic heterogeneity between fast water-rich and slow DES-rich layers. ${ }^{63}$ This hypothesis is supported by the markedly greater jump in shear force when stepping between layers seen for $30 \mathrm{wt} \%$ water compared to $50 \mathrm{wt} \%$ water, where the step in $F_{\mathrm{S}}$ is indicative of a liquid-to-solid phase transition. ${ }^{24}$ The $30 \mathrm{wt} \%$ water sample produces a larger initial elastic regime (up to the yield point), followed by plastic deformation during sliding. The $50 \mathrm{wt} \%$ has a similar normal force but a weaker solid-like response to shear, which can be attributed to a different (likely less ordered) in-layer structure for $50 \mathrm{wt} \%$ water with higher fluidity.

\section{Conclusions}

In this paper, we have presented a detailed study of the normal and shear forces acting across nanoscale films of the deep eutectic solvent ethaline, confined between charge regulated atomically flat substrates. The liquid film produces a force profile characteristic of a structural force, with clear steps corresponding to the squeeze out of molecular layers on approach and adhesive minima on retraction. Intriguingly the layer thicknesses and strengths are strongly altered by both adsorbed and added water, significantly increasing the squeezeout force.

We also detect long-ranged repulsions consistent with 'underscreening', previously only measured in ionic liquid solutions and concentrated aqueous electrolytes. The lengthscale of the repulsion is maximal for dry ethaline, and decreases with added water, unlike for conventional Debye-Hückel screening.

The frictional response of the confined film was also measured as a function of normal force, yielding a quantised frictional response with a strong dependence on water content. Remarkably we report a re-entrant superlubric regime with water content, where dry ethaline and $50 \mathrm{wt} \%$ water display superlubric properties even at high compression, whereas humid ethaline and $30 \mathrm{wt} \%$ water have a quantised frictional response with non-superlubric friction coefficients comparable 
to conventional ILs. Friction coefficients compare favourably to previously reported values, and the lubrication provided even up to $50 \mathrm{wt} \%$ water supports the previous assertion that DESs could find use as marine lubricants.

This work highlights the interplay of interfacial charge and nanoscale structure, and the role of water in modifying the influence of both. At the nanoscale liquid structure is not homogeneous, and the amphiphilicity of molecules at the local level can have dramatic consequences for structure and dynamic properties, as recorded here for the lubrication of shearing surfaces at the nanoscale. Future work will explore other DESs, as well as the role of additives in their structural and frictional properties in confinement.

\section{Conflicts of interest}

There are no conflicts to declare.

\section{Acknowledgements}

The authors thank Charles Monroe and Andrew Wang for assistance with Karl Fischer titration measurements. Funding is gratefully acknowledged from The European Research Council (under Starting Grant No. 676861, LIQUISWITCH).

\section{Notes and references}

1 A. P. Abbott, G. Capper, D. L. Davies, H. L. Munro, R. K. Rasheed and V. Tambyrajah, Chem. Commun., 2001, 2010-2011.

2 E. L. Smith, A. P. Abbott and K. S. Ryder, Chem. Rev., 2014, 114, 11060-11082.

3 A. P. Abbott, G. Capper, D. L. Davies, R. K. Rasheed and V. Tambyrajah, Chem. Commun., 2003, 70-71.

4 A. Paiva, R. Craveiro, I. Aroso, M. Martins, R. L. Reis and A. R. C. Duarte, ACS Sustainable Chem. Eng., 2014, 2, 1063-1071.

5 Y. Dai, J. van Spronsen, G. J. Witkamp, R. Verpoorte and Y. H. Choi, Anal. Chim. Acta, 2013, 766, 61-68.

6 R. Li, Q. Chu and J. Liang, RSC Adv., 2015, 5, 44933-44942.

7 H. Mou, J. Wang, D. Zhang, D. Yu, W. Chen, D. Wang and T. Mu, J. Mater. Chem. A, 2019, 7, 5719-5725.

8 A. Rajawat, S. Khandelwal and M. Kumar, $R S C A d v ., 2014,4$, 5105-5112.

9 A. E. Daz-Álvarez, J. Francos, B. Lastra-Barreira, P. Crochet and V. Cadierno, Chem. Commun., 2011, 47, 6208-6227.

10 A. P. Abbott, P. M. Cullis, M. J. Gibson, R. C. Harris and E. Raven, Green Chem., 2007, 9, 868-872.

11 O. S. Hammond, D. T. Bowron and K. J. Edler, Green Chem., 2016, 18, 2736-2744.

12 O. S. Hammond, D. T. Bowron and K. J. Edler, Angew. Chem., Int. Ed., 2017, 56, 9782-9785.

13 Z. Chen, B. McLean, M. Ludwig, R. Stefanovic, G. G. Warr, G. B. Webber, A. J. Page and R. Atkin, J. Phys. Chem. C, 2016, 120, 2225-2233.
14 O. S. Hammond, H. Li, C. Westermann, A. Y. Al-Murshedi, F. Endres, A. P. Abbott, G. G. Warr, K. J. Edler and R. Atkin, Nanoscale Horiz., 2019, 4, 158-168.

15 Z. Chen, M. Ludwig, G. G. Warr and R. Atkin, J. Colloid Interface Sci., 2017, 494, 373-379.

16 R. Pashley and J. Israelachvili, Colloids Surf., 1981, 2, 169-187.

17 W. J. Bartz, Tribol. Int., 1998, 31, 35-47.

18 A. Syahir, N. Zulkifli, H. Masjuki, M. Kalam, A. Alabdulkarem, M. Gulzar, L. Khuong and M. Harith, J. Cleaner Prod., 2017, 168, 997-1016.

19 S. D. Lawes, S. V. Hainsworth, P. Blake, K. S. Ryder and A. P. Abbott, Tribol. Lett., 2010, 37, 103-110.

20 I. Garcia, S. Guerra, J. de Damborenea and A. Conde, Lubricants, 2019, 7, 37.

21 A. P. Abbott, E. I. Ahmed, R. C. Harris and K. S. Ryder, Green Chem., 2014, 16, 4156-4161.

22 E. I. Ahmed, A. P. Abbott and K. S. Ryder, AIP Conf. Proc., 2017, 1888, 020006.

23 U. Raviv, P. Laurat and J. Klein, J. Chem. Phys., 2002, 116, 5167-5172.

24 J. Klein and E. Kumacheva, J. Chem. Phys., 1998, 108, 6996-7009.

25 S. Perkin, L. Crowhurst, H. Niedermeyer, T. Welton, A. M. Smith and N. N. Gosvami, Chem. Commun., 2011, 47, 6572-6574.

26 S. Perkin, T. Albrecht and J. Klein, Phys. Chem. Chem. Phys., 2010, 12, 1243-1247.

27 F. Endres, N. Borisenko, S. Z. El Abedin, R. Hayes and R. Atkin, Faraday Discuss., 2012, 154, 221-233.

28 O. Werzer, E. D. Cranston, G. G. Warr, R. Atkin and M. W. Rutland, Phys. Chem. Chem. Phys., 2012, 14, 5147-5152.

29 A. M. Smith, M. A. Parkes and S. Perkin, J. Phys. Chem. Lett., 2014, 5, 4032-4037.

30 A. M. Smith, K. R. Lovelock, N. N. Gosvami, T. Welton and S. Perkin, Phys. Chem. Chem. Phys., 2013, 15, 15317-15320.

31 R. Lhermerout and S. Perkin, Phys. Chem. Chem. Phys., 2020, 22, 455-466.

32 J. Sweeney, F. Hausen, R. Hayes, G. B. Webber, F. Endres, M. W. Rutland, R. Bennewitz and R. Atkin, Phys. Rev. Lett., 2012, 109, 155502.

33 H. Li, R. J. Wood, M. W. Rutland and R. Atkin, Chem. Commun., 2014, 50, 4368-4370.

34 S. Perkin, Phys. Chem. Chem. Phys., 2012, 14, 5052-5062.

35 O. Y. Fajardo, F. Bresme, A. A. Kornyshev and M. Urbakh, J. Phys. Chem. Lett., 2015, 6, 3998-4004.

36 R. Lhermerout, C. Diederichs and S. Perkin, Lubricants, 2018, 6, 9.

37 R. D. Shannon, Acta Crystallogr., Sect. A: Cryst. Phys., Diffr., Theor. Gen. Crystallogr., 1976, 32, 751-767.

38 R. Lhermerout and S. Perkin, Phys. Rev. Fluids, 2018, 3, 014201.

39 A. Pandey, R. Rai, M. Pal and S. Pandey, Phys. Chem. Chem. Phys., 2014, 16, 1559-1568.

40 A. R. Harifi-Mood and R. Buchner, J. Mol. Liq., 2017, 225, 689-695.

41 A. M. Smith, A. A. Lee and S. Perkin, J. Phys. Chem. Lett., 2016, 7, 2157-2163. 
42 A. A. Lee, C. S. Perez-Martinez, A. M. Smith and S. Perkin, Faraday Discuss., 2017, 199, 239-259.

43 A. A. Lee, C. Perez-Martinez, A. M. Smith and S. Perkin, Phys. Rev. Lett., 2017, 119, 026002.

44 J. Israelachvili, Y. Min, M. Akbulut, A. Alig, G. Carver, W. Greene, K. Kristiansen, E. Meyer, N. Pesika and K. Rosenberg, et al., Rep. Prog. Phys., 2010, 73, 036601.

45 A. M. Smith, J. E. Hallett and S. Perkin, Proc. Natl. Acad. Sci. U. S. A., 2019, 116, 25418-25423.

46 R. Lhermerout and S. Perkin, 2020, arXiv preprint arXiv:2001.01090.

47 A. Gooneie, T. E. Balmer and M. Heuberger, Phys. Rev. Res., 2020, 2, 022026.

48 H. K. Christenson and R. G. Horn, J. Colloid Interface Sci., 1985, 103, 50-55.

49 M. V. Fedorov and A. A. Kornyshev, Chem. Rev., 2014, 114, 2978-3036.

50 C. Merlet, C. Péan, B. Rotenberg, P. A. Madden, P. Simon and M. Salanne, J. Phys. Chem. Lett., 2013, 4, 264-268.

51 T. Cremer, M. Stark, A. Deyko, H.-P. Steinrück and F. Maier, Langmuir, 2011, 27, 3662-3671.

52 R. M. Espinosa-Marzal, A. Arcifa, A. Rossi and N. D. Spencer, J. Phys. Chem. Lett., 2014, 5, 179-184.

53 K. Tomita, M. Mizukami, S. Nakano, N. Ohta, N. Yagi and K. Kurihara, Phys. Chem. Chem. Phys., 2018, 20, 13714-13721.
54 M. H. Mamme, S. L. Moors, H. Terryn, J. Deconinck, J. Ustarroz and F. De Proft, J. Phys. Chem. Lett., 2018, 9, 6296-6304.

55 M. H. Mamme, S. L. Moors, E. A. Mernissi Cherigui, H. Terryn, J. Deconinck, J. Ustarroz and F. De Proft, Nanoscale Adv., 2019, 1, 2847-2856.

56 C. Merlet, D. T. Limmer, M. Salanne, R. Van Roij, P. A. Madden, D. Chandler and B. Rotenberg, J. Phys. Chem. C, 2014, 118, 18291-18298.

57 H. Zhou, M. Rouha, G. Feng, S. S. Lee, H. Docherty, P. Fenter, P. T. Cummings, P. F. Fulvio, S. Dai, J. McDonough, V. Presser and Y. Gogotsi, ACS Nano, 2012, 6, 9818-9827.

58 A. M. Smith, K. R. Lovelock and S. Perkin, Faraday Discuss., 2013, 167, 279-292.

59 H.-W. Cheng, J.-N. Dienemann, P. Stock, C. Merola, Y.-J. Chen and M. Valtiner, Sci. Rep., 2016, 6, 30058.

60 C. S. Perez-Martinez and S. Perkin, Langmuir, 2019, 35, 15444-15450.

61 A. M. Smith, K. R. Lovelock, N. N. Gosvami, P. Licence, A. Dolan, T. Welton and S. Perkin, J. Phys. Chem. Lett., 2013, 4, 378-382.

62 C. R. Ashworth, R. P. Matthews, T. Welton and P. A. Hunt, Phys. Chem. Chem. Phys., 2016, 18, 18145-18160.

63 Faraday Discuss., 2013, 167, 347-370, (General Discussion). 